\title{
THE MINOR EPILEPSIES OF CHILDHOOD
}

\author{
By NeIl Gordon, M.D.Edin., M.R.C.P., M.R.C.P.E. \\ Senior Registrar to the Department of Neurology, St. Mary's Hospital, London W.2.
}

\section{Introduction}

There is no doubt that children suffering from epilepsy tend to manifest its minor forms with greater frequency than adults. However, before considering this problem further, it is essential to define the terms to be used, as a certain amount of confusion has arisen as the result of clinicians and electroencephalographists sometimes using the same terms to refer to attacks of a different nature. No classification of the epilepsies is entirely satisfactory but it seems reasonable to refer to all seizures, other than generalized convulsions or grand mal, as minor epileptic fits. Petit mal, when the term is unqualified, should only be used when referring to those attacks which appear to be of diencephalic origin and are associated with outbursts of three cycle per second spike and wave activity in the electroencephalogram. Symonds (1955) prefers to describe them as minimal seizures or central epilepsy, as he maintains that petit mal has lost its purely clinical meaning and is a definition in terms of the electroencephalogram. This group may be further subdivided, a number of the patients suffering from attacks of this kind being grouped under the term pyknolepsy. This matter will be discussed in detail later, but suffice it to say at the moment that the distinction proposed is based not only on a number of clinical features but on certain electroencephalographic findings as well.

Seizures defined as petit mal include the familiar brief lapse of consciousness and also the myoclonic attack consisting of involuntary jerks of the muscles. It is almost certain that there is a third type in which the patient falls to the ground without loss of consciousness. Lennox (1945) has referred to this as an akinetic attack, but Gastaut (1954) does not consider that it is a separate entity and claims it is due to a sudden involvement of the lower limbs by a myoclonic jerk. It is generally assumed that these fits, included under the term petit mal, are the result of some functional disturbance and are not caused by any demonstrable alteration of structure. However, attacks which are clinically indistinguishable cer- tainly can occur after an acquired lesion, and, fo $\overrightarrow{\vec{w}}$ this reason alone, it seems justifiable to use the term petit mal in the manner suggested, coms bining both clinical and electroencephalographie criteria in the definition.

It is then possible to refer to such attacksis when they are acquired, as symptomatic petit male In this group, as will be discussed later, the history and examination will show evidence that the patient is suffering from the results of organic brain damage in addition to epilepsy. This damage is most commonly of a diffuse nature such as that due to a lipoidosis or a subacut encephalitis of the Dawson and van Bogaert type

Focal epileptic attacks do occasionally occurnim children, and these, as in adults, most frequenily arise from the temporal lobes. However, ow to the restricted capacity of a child to describe he odd features that often characterize this type of seizure (Gordon, 1957), it may not be possible ort clinical grounds to be certain if they really are of cortical origin. The impairment of consciousnes? may be very brief and, if this is unassociated witlp any remembered symptoms, these 'pseudo $\overrightarrow{\overrightarrow{0}}$ lapses' of temporal lobe epilepsy may easily bẻ confused with petit mal. Recent work (Gastaut 1954) has suggested that the term focal epilepsy. for this type of attack is inappropriate, implying that the fits arise in a restricted area of the cerebrat. cortex. It may be better to refer to them as partial epilepsy as there is considerable evidence that they do not develop in cortical areas or sub윽 cortical areas alone but in the sectors consisting of the two closely interconnected in both directions.

\section{Petit Mal}

Epileptic attacks of this kind undoubtedly occuis more frequently than any others during childhoof and they most often take the form of an inter ruption of consciousness, myoclonic or akinetie seizures being rarer. If the term petit $\mathrm{mal}$ is use in the restricted sense suggested, it is assumed tha既 these fits do not arise from any demonstrable lesion and in the majority of patients suffering 
with petit mal there is certainly nothing in the history or an examination to suggest the presence of any structural defect. There is, however, considerable evidence that the disturbance of function underlying this type of epilepsy arises within the diencephalon (Jasper and Droogleever-Fortuyn, I947), and, whatever its nature, it does render these patients unduly liable to seizures and undoubtedly in many cases it is transmitted as an hereditary factor. The fits, however frequent they may have been, tend to cease as the child grows older, although occasionally in adult life the patient may continue to suffer from grand mal, the minor seizures stopping altogether or only occurring with increasing rarity.

Although considerable stress has been laid on the electroencephalographic findings in the diagnosis of petit mal, the clinical features of the attacks are often typical enough for this to be made with confidence without further investigations being needed. However, seizures of a different kind can cause what appears to be only an interruption of consciousness so that the finding of three-per-second spike and wave activity in the electroencephalogram will undoubtedly assist in confirming this diagnosis.

As with any other investigation, the electroencephalographic findings cannot be taken as the only criteria of diagnosis and must be combined with the clinical features of the attacks, and only when both of these are typical can the diagnosis be accepted without further question. This combination of clinical and electroencephalographic evidence should dispel many of the criticisms of this definition of petit mal and, in any case in which there are unusual features, the diagnosis should not be made at least until further investigations and perhaps the passage of time have established the nature of the fits beyond all reasonable doubt.

Unusual types of seizures have been recorded under the heading of petit mal or centrencephalic epilepsy, and, to account for these, it has been suggested that there may be an asymmetrical and restricted spread by continuity of the epileptic discharge in the central areas of the brain, followed by projection of the discharge to a restricted area of the cortex in one hemisphere. This would then lead to an attack mimicking a focal cortical seizure (Howell, I955).

If the outbursts of spike and wave activity in the electroencephalogram are in any way atypical, they cannot be accepted as pathognomonic of petit mal. This applies both to the frequency of the abnormal waves and to their symmetry. As will be discussed later, spike and wave activity occuring at a frequency of less than three cycles per second has an entirely different significance; also, if these discharges showed a consistent asymmetry, it would have to be considered whether they could be secondary to a focal epileptogenic lesion in some part of the cerebral cortex. Asymmetrical discharges of this type may turn out to be of no particular significance, but they must always be viewed with suspicion as the spike and wave complex is undoubtedly a characteristic response of the infantile cerebrum to epileptogenic lesions of varying situation (Calderon and Paal, 1957).

There is one type of focal epilepsy that is particularly liable to cause difficulty in diagnosis, even in the older age groups. If the attack starts on the medial surface of the cerebral hemisphere, the only electroencephalographic abnormality may be generalized outbursts of spike and wave activity (Tükel and Jasper, 1954). However, the complexes are usually asymmetrical, irregular in form and of a frequency varying between two and twoand-a-half cycles per second. In explanation of these generalized abnormalities arising from focal cortical lesions it seems reasonable to suppose that the epileptic discharge, originating in the parasagittal areas, fires into a centrally-placed region in the diencephalon, from whence it is relayed back to the cortex on both sides (Penfield and Jasper, 1947). Such fits are rare, but should be considered when the spike and wave discharges are atypical and are associated with clinical manifestations unlike those of petit mal. These may include periods of confusion and automatic behaviour, adversive tonic-clonic convulsions, sensations referred to the head and throat, arrest of speech and forced thinking.

An example of such a diagnostic problem has been given by Bates, Cobb and Williams (1956). A child of twelve had been suffering for some years from grand mal and outbursts of aggressive behaviour. All investigations, which included airencephalography and cerebral arteriography, were normal except from the electroencephalogram. This showed frequent bilateral sharp and slow waves complexes in the frontal regions, but there were slight and consistent differences on the two sides. As the child was quite unmanageable to the extent of having to be admitted to an adult mental hospital, it was decided on the electroencephalographic evidence alone to turn a frontal flap. A large angiomatous malformation with evidence of previous haemorrhage and thrombosis was found, and after this had been removed there was sufficient improvement for the patient to return to an ordinary school.

It has also been maintained that confirming the diagnosis of petit mal by finding typical spike and wave discharges in the electroencephalogram may be misleading, because they sometimes occur in 
the records of patients suffering only from grand mal (Jasper and Kershaw, r94I). This does not seem to be a justifiable criticism, as the same could be said of the occasional, well-marked focal abnormalities in the electroencephalograms of patients with only grand mal; the spread of the discharge being so rapid that there is never any clinical evidence of the focal origin of the attacks. In either case it has been the electroencephalogram which has provided evidence as to where the epilepsy has originated from, grand mal being the end result of any such disturbance.

Petit mal attacks may occur with a frequency of several hundred a day, but, just as in other types of epilepsy, recovery. may not be complete between each individual seizure and then the patient can be said to be in petit mal status. This manifests itself clinically by periods of confusion and automatic behaviour and, if it is not realized that the child is an epileptic, a diagnosis of a behaviour disorder may be made. 'Status' of this kind is, undoubtedly, commoner in symptomatic petit mal than in the 'ideopathic' variety.

\section{Pyknolepsy}

Anyone who has treated a number of children with petit mal will have been struck by the fact that the response to treatment may vary widely. For instance, one such child, when started on Troxidone, will cease to have attacks, while another with a somewhat similar history will not respond at all. When reviewing the electroencephalograms of these children it has been noticed that although all of them might show outbursts of spike and wave activity only some would manifest runs of occipital delta activity (Elston, Gordon and Cobb, 1956). This rhythm was usually at a rate of three cycles per second, was often of high voltage, was strikingly blocked by eye opening and was increased in continuity and amplitude by overbreathing (Cobb, 1954). When the histories of the group of children showing these occipital delta rhythms in their encephalograms were analysed and compared with those of the remaining children with petit mal, certain differences were discovered. Their fits started at an earlier age, they had fewer major attacks but more frequent minor ones, and the incidence of epilepsy in their families was higher. However, the finding which differentiated the two groups more than any other was the response to treatment. This can only be assessed over a reasonably long period, as petit mal attacks frequently stop and start for no definite reason and, if this fact is not taken into account, unjustifiable credit may be given to a particular therapy. The patients showing occipital delta activity were the ones who, with few exceptions, had never shown conclusive evi- dence that treatment had affected the frequency气 of their fits to any marked degree. Some of them 3 seemed to have obtained temporary benefit, but it $\stackrel{\mathbb{Q}}{\stackrel{\mathbb{Q}}{2}}$ was commonplace for these patients to have beenc. given several different types of drugs in an effort $\vec{F}$ to control their seizures. In contrast to this the control group of patients with petit mal usually? presented no particular therapeutic problem, $\frac{\overline{\bar{N}}}{\overline{\mathrm{T}}}$ especially when they were given troxidone or one $\vec{\sigma}_{\vec{D}}$ of the other oxozalidine-dione derivatives. There- $\varrho$ fore, although no adequate explanation can bes given for the presence of these delta waves in the $-\vec{\circ}$ post-central regions, the finding of them may beof prognostic significance and suggest that the $\vec{\omega}$ child's fits may respond poorly to treatment.

The natural history of the illness in the occipital delta group was closely akin to that of pyknolepsy as it was originally defined by Friedmann (1906), $\rightarrow$ Sauer (1912) and Adie (1924). This syndrome was described as occurring in childhood andor adolescence and consisting of very frequent minor attacks, even as many as a hundred or more a day, only lasting for a few seconds at a time. These seizures started suddenly between the ages of four $\overrightarrow{c s}$ and twelve, were of uniform severity, might last for a few weeks or for several years, and were un- $\overrightarrow{\mathbb{\Phi}}$ influenced by anti-convulsant remedies. They did 3 not impede normal mental and physical develo ment and ultimately ceased spontaneously, newr to return. However, Adie (1924) considered that if the patient developed grand $\mathrm{mal}$ the prognossts was altered.

It has been suggested that the term pyknolepsys should be dropped (Williams, 1950), and that there is no justification for separating a special group? from the general run of children suffering from?

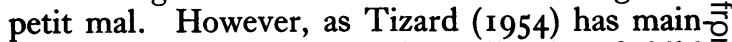
tained, the so-called ideopathic epilepsies of child 3 hood may well be separate disease entities with similar symptoms and he would oppose any? tendency to group them together in case it should? slacken the search for the underlying pathology.

The occurrence of occipital delta rhythms in the electroencephalograms of some patients with petit mal suggests that the disturbance of function? underlying this type of epilepsy may indeed not be uniform in its nature. This is supported by the slight variations in the clinical behaviour of the occipital delta group as exemplified by the descriptions of pyknolepsy, particularly as far aş the response to treatment is concerned. These्t electroencephalographic findings may be helpfuR when planning treatment as, although it wilw always be justifiable to try the effect of variouse drugs and combination of drugs, a failure to control the fits after a reasonable period of observa tion may lead to a decision to abandon them en:tirely. This will avoid submitting these patients 
to unnecessary risks associated with such drugs as troxidone, apart from the fact that the general sense of well-being is often improved when treatment is stopped.

\section{Symptomatic Petit Mal}

It has been stated that the diagnosis of "petit mal' assumes the absence of any discoverable structural lesion. However, it seems possible that an injury to the diencephalon early in life might cause a similar predisposition to minor seizure of this type, which was not constitutional. Jasper and Kershaw (194I) in a series of patients showing three-per-second spike and wave activity in their electroencephalograms reported that, in $2 \mathrm{I}$ per cent. of them, there was a previous history of cerebral trauma either at birth or soon afterwards, and, in 9 per cent., a history of various types of infection which might have involved the central nervous system. From this evidence they deduced that this type of epilepsy could be acquired. Gastaut (1954) has also stressed that brief interruptions of consciousness may be caused by a lesion of the diencephalon resulting from 'encephalitis.' He maintains that these ' lapses' following such an illness are as common as symptomatic bilateral myoclonus, although the latter has received so much more attention in the literature. However, the myoclonus is usually associated with definite evidence of structural damage, which may include the cerebellum as well as the cerebrum (Unerricht, I89I; Hunt, I92 I).

It is impossible to prove for certain that these patients are suffering from symptomatic epilepsy, as physical examination and investigations will show no definite evidence of cerebral damage. On the other hand some patients whose minor fits consist of brief interruptions of consciousness, indistinguishable from those of petit mal as already defined, will reveal definite evidence of structural disease of the central nervous system. Their attacks will usually be accompanied by electroencephalographic changes consisting of generalized outbursts of spikes or slow waves or the combination of the two in ragged complexes, at varying frequencies. The underlying disease is most often of a diffuse nature, such as that due to a cerebral lipoidosis or a subacute encephalitis of the Dawson and van Bogaert type. For instance, a patient who is ultimately diagnosed, perhaps only at postmortem examination, as an example of the first condition may present with frequent minor epileptic fits, soon to be followed by increasing mental and physical deterioration. Sometimes the electroencephalogram is of considerable diagnostic assistance in patients suspected of having cerebral lipoidosis or an inclusion-body encephalitis. In the former disease the encephalogram may show random generalized sharp transients on a background of slow waves (Cobb, Martin and Pampiglione, 1952), and in the latter the characteristic abnormality is the appearance of regular episodes of high-voltage slow waves at intervals of about eight seconds (Cobb and Hill, 1950).

Gibbs, Fleming and Gibbs (1954) have described the encephalographic findings in a group of children suffering from what they term 'infantile spasms ': attacks consisting of sudden jerking of the head, rolling of the eyes, upward flinging of the arms, and quivering of the entire body. These attacks might occur at extremely frequent intervals and it might be difficult to tell if consciousness was impaired or not. About a third of the children had grand mal as well. The electroencephalograms showed high-voltage random slow waves and spikes in all cortical areas and the term ' hypsarhythmia' was used to describe these changes. The clinical attacks were accompanied by outbursts of fast waves and high-voltage spikes. These children were frequently mentally retarded or showed other evidence of cerebral damage. The etiology was not known in over half of the cases, but encephalitis or birth injury were two of the commonest causes. Hess and Neuhaus (1952) have described similar electroencephalographic findings accompanying 'Blitz, Nick und Salaamkrämpfen.'

\section{Temporal Lobe Epilepsy}

Most varieties of focal or partial epilepsy are rare during childhood, as they usually arise from acquired lesions, more likely to be present the longer the patient has lived, and motor and sensory fits of this type do not usually present any particular diagnostic problems. However, seizures arising from within the temporal lobes are the commonest type of focal epilepsy at any age and, although their incidence increases markedly in adult life (Lennox, I95I) they are still a fairly frequent cause of minor epilepsy in childhood.

An adult often has difficulty enough in describing the clinical features of these attacks and a child may be able to add little information to that given by witnesses. All that may be apparent to an observer is an interruption of consciousness, perhaps associated with abnormal behaviour of some kind, so that it is not surprising that a diagnosis of ' petit mal' is made.

If these patients are closely questioned, suspicion may at least be aroused that there is more to the minor fits than just an interruption of consciousness. In fact, temporal lobe epilepsy can be exceedingly varied in the way it manifests itself, although, however complex these seizures may be, there are many features in the clinical picture sufficiently characteristic to suggest the 
correct diagnosis. The fits may consist of symptoms that would be expected to result from stimulation of this part of the cerebral cortex, and these can roughly be divided into four classes: motor, sensory, visceral, and psychic. The motor type may be exceedingly brief and consist of only a generalized tonic contraction of the muscles, in contrast to the clonic contractions sometimes associated with petit mal. The sensory type is characterized by a distortion of a normal sensation of one kind or another. For instance, the patient may complain of a strange smell or taste which is usually unpleasant, or visual or auditory hallucinations may occur. It will naturally be difficult to elicit a history of such attacks from a young child, but he may well have told his mother about them, who, until questioned by the doctor, may not have realized their significance. The commonest manifestation of an attack starting in the visceral brain, a term used to include parts of the insula, frontal and temporal lobes, is the 'epigastric aura.' Finally, the psychic group may cause greater diagnostic difficulties than any of the others. Different emotional feelings may occur, the most common of these being fear; an intense feeling of familiarity, the so-called déjà vu phenomenon may constitute the fit; or, conversely, it may consist of a feeling of strangeness. When this affects the environment, it may seem to the patient that he is asleep and dreaming. This is by no means a complete list of such manifestations and they do not always occur in isolation, but may be combined in various ways.

Any part of the cerebral cortex occupied or ' exhausted' by an epileptic discharge cannot be used for normal integrative functions, and this accounts for other features of temporal lobe epilepsy. For instance, if parts of the temporal lobe of the dominant hemisphere are inactivated in this way, it is likely that a disturbance of speech will result and it is not uncommon for transient attacks of dysphasia to occur in this type of focal epilepsy. Although the temporal lobes subserve many complex functions they do not seem to be essential for the more stereotyped ones, which probably explains the frequent association of automatism with temporal lobe attacks. During such episodes one is observing the integrated action of the brain excluding that part of the temporal lobe involved in the epileptic disturbance. The patient may perform a few confused movements which are not even purposive, or may carry out co-ordinated movements, though in such a way that even the inexpert eye can recognize that something is wrong. Smacking and licking of the lips is a common example of such attacks. However, occasionally the conduct of the patient during these periods may appear relatively normal. He may talk, walk around and even carry out skilled actions. On recovery, the patient will have noz recollection of what has happened, and this is no $\mathbb{Q}$ doubt related to the fact that parts of the temporal $c$ lobe appear to be essential to the laying down of memory patterns.

Patients with this type of epilepsy are particularly liable to disorders of emotion and behaviour. This may present a considerably greater problem than the occurrence of occasional fits and may be $\varrho$ the reason for seeking medical advice. This asso- $ळ$ ciation is so common that, if an epileptic child $\vec{\circ}$ presents with abnormalities of behaviour, the possibility that the fits are arising in the temporal $\vec{\omega}$ lobe should be seriously considered.

Although the diagnosis of temporal lobe epilepsy缥 in children may be particularly difficult, help can often be obtained from the electroencephalogram. The specific seizure discharge is the occurrence of $\omega$ well-marked focal abnormalities in the temporal or regions. These may consist of irregular slow waves or of random spikes and sharp waves. However, the record taken when the patient is 5 awake is frequently normal or it may only show a $\vec{C}$ non-specific dysrhythmia. It is then of value to $\mathbb{D}$ take a record while the patient is going to sleep or $\frac{\overrightarrow{\mathbb{D}}}{\mathrm{D}}$ during a period of deep sleep (Gibbs and Gibbs, 3 1947). Spikes and other focal abnormalities mąy appear, most frequently as the patient becomo drowsy, but sometimes only when he is asleep. On

Once the diagnosis of temporal lobe epilepsy ha been made, the implications are the same as for any other type of focal epilespy. Further investigations may well be indicated, as even in child-o hood these attacks may be the first symptom of a cerebral tumour. However, as in any other type $\stackrel{\mathbb{\Omega}}{\circ}$ of epilepsy, there will be many cases in which no $\overrightarrow{\overrightarrow{0}}$ definite underlying pathology can be demonstrated 3 although the etiology may be suggested from other evidence. For instance, a history of cerebralo trauma is not uncommon, the hippocampal gyrus 흠 being particularly liable to damage in closed head 3 . injuries. This may be due to generalized cerebral oedema causing herniation of the hippocampus through the tentorial opening. A lesion in this area may then result from interference with the blood supply. A similar mechanism possibly $\frac{\text { ? }}{2}$ operates in birth injuries and it has been suggested $\rightarrow$ that the lesion may ripen into an epileptogenic focus in later life (Earle and Penfield, 1953). N

The treatment of temporal lobe epilepsy presents particular difficulties and these fits will not usually 0 respond to the same drugs that are likely to control $\omega$ petit mal. Therefore, quite apart from its different diagnostic and prognostic implications, it is important to separate this variety of epilepsy from $\stackrel{Ð}{\Phi}$ the other minor seizures of childhood. When investigations have helped to exclude any underly- 
ing condition likely to be amenable to surgery, a decision will have to be taken on medical treatment. It is always worth while trying the effect of barbiturates, either alone or combined with phenytoin sodium. Then, if the fits are still poorly controlled, other drugs, such as methoin and phenacetylurea, may have to be given, although they carry a risk of toxic complications. Finally, if it has to be admitted that this treatment has failed and there is a well-marked electroencephalographic focus, the question of its surgical excision should be considered. In such a case, other investigations may well have been negative and even at operation no definite macroscopical evidence of a lesion may be found. However, microscopical examination of the material removed sometimes shows evidence of focal damage, such as atrophy or a small vascular anomaly.

\section{Summary}

When a diagnosis of epilepsy is made in childhood particular attention should be paid to the characteristics and pattern of any minor attacks that may have occurred, although it will not always be possible to classify these into a particular group which has an especial significance of its own. It will be found that the majority of the minor fits will consist of a brief lapse of consciousness or of myoclonic jerks. The term ' petit mal' should only be used to refer to fits of this kind, which appear to have their origin in deep, mid-line structures and are associated with three-cycle-per-second spike and wave activity in the electroencephalogram. There is a subdivision of this group, which, it is suggested, is a definite clinical entity of its own and to which the term pyknolepsy should be restricted. The minor fits of pyknolepsy have no specific characteristics except, perhaps, their tendency to occur at very frequent intervals. However, they are particularly difficult to control with anti-convulsant drugs of any kind, and it is common to find that patients suffering from pyknolepsy have been tried on numerous drugs and have been taken to see many different doctors by their distressed parents. Although the immediate prognosis is poor, the ultimate prognosis is good and the attacks frequently tend to stop at puberty or soon afterwards. The reassurance that can, therefore, be given to the parents is often of great comfort. The clinical diagnosis of attacks of this kind may only be made in retrospect, but the associated electroencephalographic changes can be of considerable help. In addition to the paroxysmal outbursts of spike and wave complexes, runs of occipital delta activity may be found in the records of these patients. It is suggested that the slight variations in the natural history of the fits and the electro- encephalographic abnormalities warrant separating, under the term pyknolepsy, a number of patients suffering from brief lapses of consciousness, as it is possible that the underlying disorder may in some way differ from that causing other attacks of a similar kind.

Brief lapses of consciousness and myoclonic jerks may be the initial manifestation of an organic disease of the brain, and these attacks may be referred to as symptomatic petit mal. The pathological process is usually found to be diffuse and degenerative, such as a cerebral lipoidosis or a subacute encephalitis. The course of the illness and the results of special investigations will often indicate the correct diagnosis. The electroencephalogram may show not only paroxysmal discharges, but a continuous background of diffuse abnormalities. The paroxysmal outbursts will not have the regular pattern associated with petit mal, but will consist of spikes or slow waves, either alone or very irregularly combined into ragged complexes at a frequency which is usually below three cycles per second. These changes may be sufficiently specific to support a particular diagnosis.

There is a group of children whose minor attacks consist not only of impaired consciousness but also of myoclonic jerks or spasms. Their electroencephalographic findings have been groupedo under the term hypsarhythmia, the abnormal spikes and slow waves being of particularly high voltage. These children show, in most cases," other evidence of damage to the central nervous system, especially mental defect, and there may be a previous history of such conditions as encephalitis or birth injury.

Focal epileptic fits are relatively uncommon during childhood, and even when they do occur may not have the same significance as in adult life. In very young children the apparent focus of origin of the seizures may change and no definite evidence of a corresponding lesion may ever be found. Another point of confusion is that in children an epileptogenic lesion in the cortex may cause a spike and wave discharge. However, this is usually well localized except in the case of lesions on the medial surface of the cerebral hemispheres, when this type of discharge may be generalized.

Although not as common as in adults, fits arising in the temporal lobes may sometimes cause diagnostic difficulties, particularly as a child will find it hard to describe the varied manifestations of this type of epilepsy. The attacks may consist of features that would be expected to occur from stimulation of this part of the cortex, such as olfactory, visual and auditory hallucinations or, because the cortex cannot be used for normal 
functions while it is occupied by an epileptic discharge, there may be evidence of the limited integration of the rest of the brain in the form of periods of ' automatism.'

The diagnosis from petit mal is of special importance as the presence of a progressive destructive lesion in the temporal lobe will have to be excluded, and if there is no indication for surgical treatment, the type of drug likely to control the seizures will be entirely different. Troxidone is one of the most successful drugs in the treatment of petit mal, while focal fits arising in the temporal lobes may respond best to one of the hydantoinate series.

Even if it is not always possible to make an exact diagnosis of the variety of epilepsy from which a particular child is suffering, a detailed study of these seizures will frequently produce a great deal of information on the various disorders that may underlie them. For instance, it seems probable that petit mal may be the result of more than one kind of disturbance of cerebral function, the similarity of the fits only being a reflection of the limited way in which a child's brain can react to such stimuli.

\section{BIBLIOGRAPHY}

ADIE, W. J. (1924), Brain, 47, 96.
BATES, J. A. V., COBB, W., and WILlIAMS, D. J. (1956), Electroenceph. clin. Neurophysiol., 8, $16 \mathrm{r}$.

CALDERON, A., and PAAL, G. (1957), Ibid., 9, 350.

COBB, W. (1954), Proc. roy. Soc. Med., 47, 846.

COBB, W., and HILL, D. (1950), Brain, 73, 392.

COBB, W., MARTIN, F., and PAMPIGLIONE, G. (1952), Ibid., 75,343 .

EARLE, K. M., and PENFIELD, W. (1953), Arch. Neur. Psychiat. (Chicago), 69, 27.

ELSTON, C., GORDON, N. S., and COBB, W. (1956), Electroenceph. clin. Neurophysiol., 68, I6r.

FRIEDMANN, M. (1906), Dtsch. Z. Nervenheilk., 30, 462.

GASTAUT, H. (1954), 'The Epilepsies,' Charles C. Thomas, Springfield, Illinois, U.S.A.

GIBBS, E. L., and GIBBS, F. A. (1947), Proc. Ass. Res. Nerv. Ment. Dis., 26, 366.

GIBBS, E. L., FLEMING, M. M., and GIBBS, F. A. (1954), Pediatrics, 13, 66.

GORDON, N. S. (1957), Postgrad. med. F., 33, 228.

HESS, R. J., and NEUHAUS, T. (1952), Arch. Psychiat. Nervenkr., 189, 37.

HOWELL, D. A. (1955), Brain, 78, 199.

HUNT, R. (1921), Ibid., 44, 490.

JASPER, H., and DROOGLEEVER-FORTUYN, J. (1947), Proc. Ass. Res. Nerv. Ment. Dis., 26, 272.

JASPER, H., and KERSHMAN, J. (I94I), Arch. Neur. Psychiat. (Chicago), 45, 903

LENNOX, W. G. (1945), 7. Amer. med. Ass., 129, 1069.

LENNOX, W. G. (I951), Neurology, I, 357.

PENFIELD, W., and JASPER, H. H. (1947), Proc. Ass. Res. Nerv. Ment. Dis., 26, 252.

SAUR (1912), Mschr. Physiat. Neurol., 40, 27.

SYMONDS, C. P. (1955), Brit. med. F., i, r 235.

TIZARD, J. P. M. (1954), Proc. roy. Soc. Med., 47, 845.

TUKEL, K., and JASPER, H. H. (1955), Electroenceph. clin. Neurophysio., 4, 481.

UNVERRICHT, H. (I89r), ' Die Myoclonie,' Leipzig .

WILlIAMS, D. (1950), Brit. med. $\mathcal{F}$., i, 685.

\section{CARDIAC DISEASE}

Price 3s. 11d. post free

INTRODUCTION

Walter Somerville, M.D., M.R.C.P.

ANGIOGRAPHY

J. Norman Pattinson, M.B., B.Chir., D.M.R.D., F.F.R.

BEDSIDE DIAGNOSIS OF CONGENITAL HEART DISEASE

Walter Somerville, M.D., M.R.C.P.

SURGICAL TREATMENT OF CONGENITAL HEART DISEASE

W. P. Cleland, M.R.C.P., F.R.C.S.

PREGNANCY AND RHEUMATIC HEART DISEASE

Samuel Oram, M.D., F.R.C.P.
DRUG TREATMENT OF HYPERTENSION

E. G. McQueen, M.B., M.R.C.P., and F. H. Smirk, M.D., F.R.C.P.

TREATMENT OF BACTERIAL ENDOCARDITIS

Ian G. W. Hill, C.B.E., T.D., M.B., F.R.C.P.E., M.R.C.P., F.R.S.E

THE MANAGEMENT OF COR PULMONALE

J. F. Goodwin, M.D., M.R.C.P.

THE CARDIAC RISK IN ANAESTHESIA AND SURGERY

Graham W. Hayward, M.D., F.R.C.P.

Published by

THE FELLOWSHIP OF POSTGRADUATE MEDICINE

60, Portland Place, London, W.1 